ARQGA/1156

\title{
CORRELAÇÃO ENTRE A CONTAGEM DE PLAQUETAS NO SANGUE E O GRADIENTE DE PRESSÃO VENOSA HEPÁTICA EM PACIENTES CIRRÓTICOS
}

\author{
Sirlei DITTRICH, Angelo Alves de MATTOS, \\ Hugo CHEINQUER e Fernanda Branco de ARAÚJO
}

RESUMO - Racional - A medida do gradiente de pressão venosa hepática é o método mais utilizado para a avaliação da pressão portal. Mais recentemente, a contagem de plaquetas no sangue tem sido apontada como um marcador não-invasivo da presença de hipertensão portal. Objetivo - Correlacionar a contagem de plaquetas com os valores do gradiente de pressão venosa hepática em uma população de pacientes cirróticos. Pacientes e Métodos - Foram estudados 83 pacientes com hepatopatia crônica que realizaram estudo hemodinâmico hepático, em período de 6 anos. Os pacientes foram divididos em grupos conforme a classificação de Child-Pugh e todos realizaram endoscopia digestiva alta para constatar a presença de varizes de esôfago, assim como tiveram a contagem sérica de plaquetas determinada. Resultados - O número de plaquetas variou entre $45.000 / \mathrm{mm}^{3}$ e $389.000 / \mathrm{mm}^{3}$, com média 104.099 e desvio-padrão 58.776. O gradiente de pressão venosa apresentou média igual a 15,2 $\mathrm{mm} \mathrm{Hg}$ e desvio-padrão igual a 6,4 mm Hg, variando de 1 a 29 mm Hg. Realizou-se regressão linear simples para verificar a correlação entre o gradiente de pressão venosa e o número de plaquetas, o que permitiu constatar fraca correlação entre ambos. Embora se tenha observado menor número de plaquetas, à medida que o calibre das varizes aumentava e nos pacientes com maior grau de disfunção hepatocelular - medida pela classificação de Child-Pugh - não se encontrou significância estatística. Conclusão - A despeito de não haver demonstrado correlação estatística entre o número de plaquetas com o gradiente de pressão venosa hepática e o grau de disfunção hepatocelular, pelas tendências observadas, acredita-se que ambos os fatores podem estar implicados na patogenia da plaquetopenia em pacientes cirróticos.

DESCRITORES - Pressão na veia porta. Hipertensão portal. Contagem de plaquetas. Cirrose hepática.

\section{INTRODUÇÃO}

A hipertensão portal (HP) é uma síndrome clínica freqüente, caracterizada por aumento patológico na pressão venosa portal e formação de colaterais portossistêmicas, que desviam sangue portal para a circulação sistêmica ${ }^{(4)}$.

A avaliação da presença de HP pode ser feita através de dados clínicos e de procedimentos não-invasivos e invasivos, podendo o sistema portal ser contrastado através de várias técnicas ${ }^{(26,30,45)}$. No entanto, a medida da pressão portal é o procedimento mais apropriado para avaliar sua real elevação ${ }^{(14}$ ${ }^{19)}$. Esta pode ser determinada por métodos $\operatorname{diretos}^{(6,8,9,48)}$ ou indiretos ${ }^{(19)}$. Tendo em vista as dificuldades na realização dos métodos diretos, tem-se preferido a determinação da pressão portal através do método indireto.

O método indireto de medida utilizado, que reflete de forma fidedigna a pressão venosa portal, é a pressão venosa hepática ocluída pois, ocluindo-se um ramo da veia hepática, a pressão medida será a do espaço sinusoidal. Essa técnica foi desenvolvida por MYERS e TAYLOR ${ }^{(28)}$ e tem sido a mais utilizada para este fim. Nesse método, o ponto de referência é a pressão venosa hepática livre, medida na junção da veia hepática e cava inferior ou na veia cava inferior. A medida da pressão venosa hepática ocluída e livre e o gradiente entre essas duas pressões, denominado gradiente de pressão venosa hepática (GPVH), apesar de invasivo, é método seguro para a avaliação da pressão portal, que vem sendo utilizado há mais de $50 \operatorname{anos}^{(1,2,15,19,20,42)}$.

A medida dos níveis da pressão portal através desta técnica, pode auxiliar no diagnóstico diferencial das causas de hipertensão portal, na avaliação do risco de sangramento por ruptura de varizes gastroesofágicas, na avaliação da eficácia do tratamento medicamentoso, na profilaxia do sangramento por varizes gastroesofágicas, na decisão terapêutica em casos de ressecção hepática e na avaliação do prognóstico desses pacientes $^{(1,5,7,12,26,28,29,30,31)}$.

Apesar das indiscutíveis aplicações do GPVH, este método é pouco disponível por ser oneroso e necessitar profissionais

Curso de Pós-Graduação em Hepatologia da Fundação Faculdade Federal de Ciências Médicas de Porto Alegre - FFFCMPA, Porto Alegre, RS

Endereço para correspondência: Dra. Sirlei Dittrich - Rua Pedro Ivo, 625 - apt.601 - Monte Serrat - 90450-210 - Porto Alegre, RS. 
treinados. Além disso, apesar de ser menos agressivo do que as demais técnicas de medida direta da pressão portal, é uma técnica invasiva. Por essas razões, há o desafio de se identificar um marcador não-invasivo para a hipertensão portal ${ }^{(24)}$.

Vários autores ${ }^{(40,50)}$ sugeriram que alguns parâmetros da ultrasonografia com Doppler pudessem ter valor prognóstico e serem úteis na avaliação do risco de sangramento por varizes de esôfago. No entanto, esta técnica tem sido pouco empregada com este fim e sua utilidade clínica tem sido debatida ${ }^{(36)}$.

A endoscopia digestiva alta pode viabilizar a medida da pressão intravariceal e estimar o risco de sangramento por varizes de esôfago. Pode-se utilizar um medidor de pressão pneumático acoplado à ponta do endoscópio ou por punção direta das varizes com uma agulha de esclerose $^{(17)}$. Esses métodos são pouco utilizados em nosso meio por serem mais invasivos ou pela falta de disponibilidade dos equipamentos.

Mais recentemente, a contagem sérica das plaquetas tem sido apontada como marcador não-invasivo da presença de hipertensão $\operatorname{portal}^{(13,21,22,39)}$.

Este estudo teve como objetivo correlacionar os níveis de plaquetas no sangue com os valores do gradiente de pressão venosa hepática em uma população de pacientes cirróticos.

\section{MATERIAL E MÉTODOS}

Estudaram-se 83 pacientes com hepatopatia crônica, que realizaram estudo hemodinâmico hepático no Setor de Hemodinâmica da Irmandade da Santa Casa de Misericórdia de Porto Alegre, RS. (ISCMPA), num período de 6 anos.

O diagnóstico de cirrose foi confirmado por dados clínicos, laboratoriais e ecográficos e/ou exame anatomopatológico.

A etiologia viral da hepatopatia crônica foi definida através da realização do anticorpo para vírus da hepatite $\mathrm{C}$ e do antígeno de superfície para vírus da hepatite B. A etiologia alcoólica foi definida quando houvesse ingesta maior de $80 \mathrm{~g}$ de etanol por dia, durante período igual ou superior a $5 \operatorname{anos}^{(15,46)}$. As demais etiologias foram excluídas pela história clínica ou com exames específicos realizados nos pacientes sem evidência de etiologia alcoólica, viral ou medicamentosa.

Os pacientes foram divididos em grupos conforme a classificação de Child-Pugh ${ }^{(35)}$. Todos realizaram endoscopia digestiva alta para constatar a presença de varizes de esôfago e foram submetidos ao estudo hemodinâmico hepático para determinação do GPVH.

As varizes de esôfago foram classificadas da seguinte forma: grau 1 - varizes pequenas ( 1 a $3 \mathrm{~mm}$ ) que colabam à insuflação de ar, grau 11 - varizes moderadas (4 a $6 \mathrm{~mm}$ ) e grau 111 - varizes grandes (maiores de $6 \mathrm{~mm}$ ). O parâmetro para estas medidas foi o diâmetro de abertura da pinça de biopsia $(5 \mathrm{~mm})^{(46)}$.

A cateterização da veia hepática foi obtida através da punção da veia femoral. A pressão venosa hepática foi medida através da colocação de um cateter radiopaco na veia hepática sob controle radioscópico. Conectado a este cateter estava um fisiógrafo, que registra uma curva de pressão. A pressão venosa hepática ocluída foi mensurada com a ponta do cateter, bloqueando pequeno ramo da veia hepática. Confirmou-se a posição correta de oclusão da mesma através dos seguintes critérios, seguindo os preceitos sugeridos por GROSZMANN et al. ${ }^{(19)}$ : 1. presença de uma curva de pressão estável; 2. ausência de refluxo de material de contraste para a veia hepática, ou seja, era injetado contraste e este deveria progredir e opacificar o leito sinusoidal e não refluir; 3. queda importante da pressão na retirada do cateter, ou seja, este era tracionado e registrava-se diminuição na curva de pressão. Em geral, as medidas da pressão venosa hepática ocluída em diferentes veias hepáticas em pacientes com cirrose, são idênticas. Assim, o valor obtido inicialmente era confirmado pela oclusão do cateter em um segundo ramo da veia hepática. Se houvesse discrepância entre os dois valores, a pressão ocluída era realizada novamente numa terceira veia hepática. Este terceiro valor, usualmente idêntico a um dos dois prévios, foi usado como valor final. A pressão venosa hepática livre foi mensurada na veia hepática ou na junção da veia hepática e veia cava inferior ${ }^{(19)}$. O GPVH foi obtido através da diferença entre a pressão venosa ocluída e livre.

Os seguintes critérios foram observados como contra-indicação à realização do estudo hemodinâmico: idade menor de 18 anos, gravidez, mau estado geral, encefalopatia portossistêmica, peritonite bacteriana espontânea, hemorragia digestiva aguda, insuficiência cardíaca grau $1 \mathrm{~V}$, insuficiência respiratória grave, uso de betabloqueadores, alterações hemodinâmicas graves, atividade de protrombina abaixo de $50 \%$, contagem de plaquetas abaixo de $50.000 / \mathrm{mm}^{3}$. Ressalve-se haver estudo recente que conclui que a atividade anormal de protrombina não estava relacionada com aumento no risco de sangramento após angiografia ${ }^{(11)}$.

A não-canulação da veia hepática por problemas técnicos também se constituiu em critério de exclusão. De todos os pacientes foi exigido consentimento informado para a realização do estudo.

Todos os pacientes tiveram a contagem sérica de plaquetas determinada através do método automatizado, que fornece resultados reprodutíveis, apesar do alto custo do aparelho.

O projeto foi submetido a Comissão de Ética da Instituição (ISCMPA).

As variáveis quantitativas foram apresentadas em forma de média e desvio-padrão; as qualitativas, em forma de freqüência e proporção. Para a comparação de médias foi utilizado a análise de variância (ANOVA). Para se analisar a relação entre as variáveis quantitativas foi utilizado o coeficiente de correlação de Pearson. O nível de significância assumido foi de 0,05 .

\section{RESULTADOS}

Dos 83 pacientes, $59(71,1 \%)$ eram homens e $24(28,9 \%)$ mulheres. A idade variou de 26 a 75 anos, sendo a média de 52,9 anos, com desvio-padrão de 10,1 . Em relação à cor, 71 pacientes $(85,5 \%)$ eram brancos e $12(14,5 \%)$ pretos.

Em 25 (30,1\%) o álcool foi o único fator envolvido na etiologia da hepatopatia; em 26 (31,3\%), o álcool estava associado ao vírus $\mathrm{B}$ ou C. Em 28 (33,7\%), a etiologia foi associada ao vírus B ou C e em 4 pacientes $(4,8 \%)$, a cirrose foi considerada criptogenética.

Vinte e três pacientes $(27,7 \%)$ eram Child A, 48 (57,8\%) Child B, $11(13,3 \%)$ Child C, e em $1(1,2 \%)$ não se obteve este dado por extravio do registro.

Nove pacientes (10,8\%) apresentaram varizes de esôfago grau I, 27 $(32,5 \%)$ varizes grau II, $44(53,0 \%)$ tinham varizes grau III, 1 paciente $(1,2 \%)$ apresentou apenas varizes de fundo gástrico e em $2(2,4 \%)$ este dado não foi resgatado. Ressalve-se que ambos apresentavam sinais de hipertensão portal à ecografia.

O número de plaquetas variou entre $45.000 / \mathrm{mm}^{3}$ e $389.000 / \mathrm{mm}^{3}$, com média 104.099 e desvio-padrão 58.776. 
O gradiente de pressão venosa apresentou média igual a 15,2 mm Hg e desvio-padrão igual a 6,4 mm Hg, variando de 1 a $29 \mathrm{~mm} \mathrm{Hg}$.

Foi realizada regressão linear simples para verificar a correlação entre o GPVH e o número de plaquetas. O coeficiente de correlação de Spearman encontrado foi $\mathrm{r}=-0,246(P=0,027)$. Este resultado denota fraca correlação (pela escala sugerida por Callegari) entre as duas variáveis. $\mathrm{O}$ coeficiente de determinação $\mathrm{r}^{2}=0,0605$, permite afirmar que aproximadamente $6,1 \%$ das variações do GPVH podem ser previstas pelo conhecimento da variação do número de plaquetas. Pode-se confirmar estes achados pela dispersão dos dados observados no Gráfico 1.

GRÁFICO 1 - Dispersão para número de plaquetas e gradiente de pressão venosa

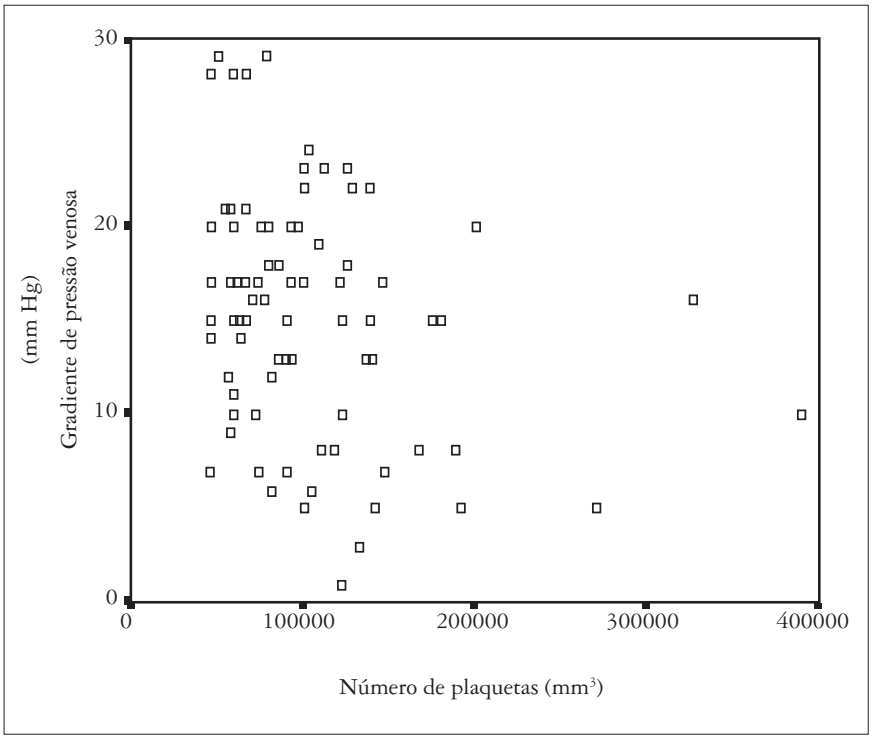

Na Tabela 1 analisa-se o número de plaquetas e o grau das varizes esofágicas.

TABELA 1 - Resultados descritivos do número de plaquetas em função do grau de varizes

\begin{tabular}{ccccc}
\hline $\begin{array}{c}\text { Grau de } \\
\text { varizes }\end{array}$ & Mínimo & Máximo & Média (DP) & Mediana (AI) \\
\hline I & $46.000 / \mathrm{mm}^{3}$ & $125.000 / \mathrm{mm}^{3}$ & $89.556(28.228)$ & $100.000(52.500)$ \\
II & $46.000 / \mathrm{mm}^{3}$ & $389.000 / \mathrm{mm}^{3}$ & $116.370(76.861)$ & $93.000(48.000)$ \\
III & $45.000 / \mathrm{mm}^{3}$ & $271.000 / \mathrm{mm}^{3}$ & $96.071(46.567)$ & $80.500(63.500)$ \\
\hline
\end{tabular}

${ }^{*}$ Kruskal-Wallis: $P=0,451$

** DP: desvio-padrão

Al: amplitude interquartilica

*** plaquetas $/ \mathrm{mm}^{3}$

Não foi possível estabelecer padrão de comportamento do número de plaquetas em função do grau de varizes a partir das médias. Pôdese observar que o número médio de plaquetas aumenta e depois diminui, à medida que é maior o grau de varizes. As diferenças entre as médias não puderam ser consideradas estatisticamente significativas a partir do teste de Kruskal-Wallis. Não obstante, ao se observar as medianas (dada a assimetria da distribuição do número de plaquetas), verifica-se número de plaquetas menor à medida que o grau de varizes aumenta.
No Gráfico 2 pode-se observar o comportamento irregular do número de plaquetas em relação ao grau das varizes de esôfago.

GRÁFICO 2 - "Boxplot" do número de plaquetas em função do grau de varizes

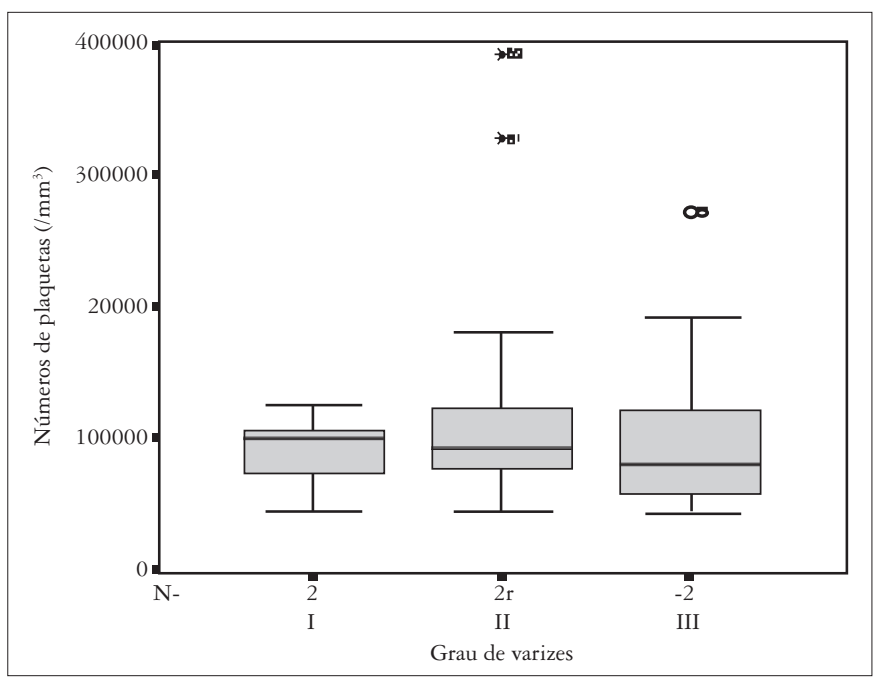

Ao se verificar a média do número de plaquetas em relação à classificação de Child-Pugh, não se identificou diferença estatística entre os grupos $(P=0,844)$. Os resultados podem ser observados na Tabela 2 .

TABELA 2 - Número de plaquetas em relação à classificação de Child-Pugh

\begin{tabular}{ccccc}
\hline Child-Pugh & Mínimo & Máximo & Média (DP) & Desvio-padrão \\
\hline A & $46.000 / \mathrm{mm}^{3}$ & $125.000 / \mathrm{mm}^{3}$ & 114.591 & 74.781 \\
B & $46.000 / \mathrm{mm}^{3}$ & $389.000 / \mathrm{mm}^{3}$ & 100.688 & 53.741 \\
C & $45.000 / \mathrm{mm}^{3}$ & $271.000 / \mathrm{mm}^{3}$ & 99.200 & 46.720 \\
\hline * Kruskallis: $P=0.844$ & & &
\end{tabular}

** plaquetas $/ \mathrm{mm}^{3}$

Não se identificou, também, associação significativa entre a classificação de Child-Pugh e o GPVH. Como podem ser observadas na Tabela 3, as médias do GPVH não diferiram de forma significativa, quando analisadas de acordo com a classificação de Child-Pugh (análise de variância ANOVA, $P=0,744)$.

TABELA 3 - GPVH para cada classificação de Child-Pugh

\begin{tabular}{ccccc}
\hline $\begin{array}{c}\text { GPVH } \\
\text { Child-Pugh }\end{array}$ & Mínimo & Máximo & Média & Desvio-padrão \\
\hline A & $1 \mathrm{~mm} \mathrm{Hg}$ & $28 \mathrm{~mm} \mathrm{Hg}$ & 14,43 & 6,19 \\
B & $3 \mathrm{~mm} \mathrm{Hg}$ & $29 \mathrm{~mm} \mathrm{Hg}$ & 15,67 & 6,17 \\
C & $6 \mathrm{~mm} \mathrm{Hg}$ & $29 \mathrm{~mm} \mathrm{Hg}$ & 14,91 & 8,15 \\
\hline *Kruskal-Wallis: $P=0,844$ & & &
\end{tabular}

Avaliaram-se a seguir, os pacientes segundo o grau de falência hepatocelular e os níveis da pressão portal, correlacionando-os com a contagem das plaquetas.

Quando se agruparam os pacientes com Child A e com GPVH menor que $12 \mathrm{~mm} \mathrm{Hg}$, encontrou-se número médio de plaquetas igual $179.500 / \mathrm{mm}^{3}$ e desvio-padrão igual a $108.128 / \mathrm{mm}^{3}$, mediana igual a 144.000/ $\mathrm{mm}^{3}$; para os paciente com Child C e GPVH maior que 12 $\mathrm{mm} \mathrm{Hg}$, número médio de plaquetas igual a $98.800 / \mathrm{mm}^{3}$, com desvio- 
padrão igual a $48.891 / \mathrm{mm}^{3}$ e mediana igual a $85.500 / \mathrm{mm}^{3}$. Não se verificou diferença estatisticamente significativa entre as médias pelo teste Kruskal-Wallis, $P=0,208$.

\section{DISCUSSÃO}

Em indivíduos normais, o GPVH varia de 1 a $5 \mathrm{~mm} \mathrm{Hg}$, sendo considerado haver hipertensão portal quando os níveis estiverem acima desses valores ${ }^{(1,10)}$. Em pacientes hepatopatas crônicos, o GPVH pode variar de 10 a $30 \mathrm{~mm} \mathrm{Hg}$, a despeito de se poder observar valores inferiores a $10 \mathrm{~mm} \mathrm{Hg}^{(1,2,14)}$. No presente estudo, a média do GPVH foi de $15,26 \mathrm{~mm} \mathrm{Hg}$. Apesar das variações observadas, na dependência da casuística avaliada, os valores encontrados são coincidentes com os da literatura ${ }^{(24)}$.

A elevação do GPVH está relacionada à presença de varizes de esôfago e ainda, prognostica o maior risco de sangramento nos pacientes com gradiente acima de $12 \mathrm{~mm} \mathrm{Hg}(1,12,18,27,29,38,44)$. Assim como a presença das varizes de esôfago, a plaquetopenia é outro achado freqüente nos pacientes hepatopatas crônicos ${ }^{(25)} \mathrm{e}$ tem sido associada a dois fatores ${ }^{(33)}$ : o primeiro relaciona-se ao hiperesplenismo decorrente de esplenomegalia na hipertensão portal. O baço seqüestra continuamente um terço das plaquetas circulantes, fazendo com que a esplenomegalia, particularmente quando causada por congestão passiva ou por aumento da pressão venosa portal, aumente a fração de plaquetas retidas nos sinusóides esplênicos. O hiperesplenismo parece ser a causa mais freqüente de plaquetopenia associada à cirrose hepática e hipertensão portal ${ }^{(16)}$. O segundo mecanismo seria relacionado à diminuição na produção de trombopoietina, hormônio produzido pelos hepatócitos, que regula o desenvolvimento do megacariócito. Na cirrose, pela redução da massa de hepatócitos funcionantes, ocorreria redução da trombopoiese na medula óssea, levando à plaquetopenia no sangue periférico ${ }^{(21,22,32)}$. Ressalve-se que nem todos os autores concordam com esta assertiva ${ }^{(41)}$.

Sendo a hipertensão portal um dos mecanismos a explicar a plaquetopenia, esta poderia ser o índice indireto de uma de suas mais temíveis complicações: a presença de varizes de esôfago. Assim, na tentativa de tornar a avaliação do paciente cirrótico cada vez menos invasiva, alguns autores ${ }^{(37,43,49)}$ têm desenvolvido estudos correlacionando a contagem sérica de plaquetas com a presença de varizes de esôfago. Esses estudos demonstraram que quanto menor o número de plaquetas, mais calibrosas as varizes esofagianas. SCHEPIS et al. ${ }^{(37)}$, utilizando ponto de corte de 100.000 plaquetas $/ \mathrm{mm}^{3}$ associado a outros achados como tempo de protrombina e o calibre da veia porta na ecografia, concluíram que a endoscopia digestiva alta para diagnóstico de varizes esofagianas só deveria ser realizada nos pacientes cirróticos compensados que apresentassem plaquetas em números inferiores a $100.000 / \mathrm{mm}^{3}$, tempo de protrombina menor que $70 \% \mathrm{e}$ calibre da veia porta maior que $13 \mathrm{~mm}$ à ecografia. Esse resultado vai ao encontro de estudo recente realizado por THOMOPOULOS et al. ${ }^{(43)}$ que, avaliando uma população de 184 cirróticos, sugeriram que a endoscopia para rastreamento de varizes de esôfago poderia ser evitada quando as plaquetas fossem superiores a $118.000 / \mathrm{mm}^{3} \mathrm{e}$ não houvesse esplenomegalia ou ascite, sendo esses fatores de risco independentes para a presença de varizes de grande calibre. ZAMAN et al. ${ }^{(49)}$, discriminaram um valor de plaquetas de $88.000 / \mathrm{mm}^{3}$ para a presença de varizes calibrosas.
No presente estudo tentou-se associar a presença e o grau das varizes de esôfago com a contagem sérica de plaquetas, não havendo diferença estatisticamente significativa entre os grupos. No entanto, quando avaliada a mediana, verificou-se menor número de plaquetas à medida que o grau de varizes aumenta. É importante ressaltar que não houve correlação estatisticamente significativa entre a contagem sérica de plaquetas e os valores do GVHP na população estudada. Desta forma, parece que a plaquetopenia, quando avaliada de forma isolada, não poderia ser vista como fator preditivo de hipertensão portal. Quando se revisou a literatura, na tentativa de se estabelecer parâmetros comparativos com os resultados aqui apresentados, não foram encontrados estudos avaliando a contagem de plaquetas com o GPVH.

Na prática clínica, é comum utilizar-se a classificação de Child-Pugh para se avaliar o prognóstico de pacientes cirróticos. Mais recentemente, alguns estudos têm relacionado a medida do GPVH com o prognóstico dos mesmos pacientes ${ }^{(1,23)}$. Le MOINE et al. ${ }^{(23)}$ estudaram 957 pacientes cirróticos e relacionaram o GPVH à classificação de Child-Pugh, concluindo que, independente da presença de varizes de esôfago, o GPVH foi maior nos pacientes Child C, quando comparados aos com Child A ou B. Em outro estudo, que avaliou os fatores preditivos para sangramento por varizes de esôfago e sobrevida em pacientes cirróticos de etiologia alcoólica, STANLEY et al. ${ }^{(41)}$ também observaram correlação significativa entre a classificação de Child-Pugh e o GPVH. Na presente série, a média do GPVH foi similar nos pacientes Child A, B e C.

Da mesma forma como se considerou o GPVH, não se encontrou na literatura estudos que avaliassem a correlação entre a média de plaquetas e a função hepática dos pacientes cirróticos através da classificação de Child-Pugh. Os estudos avaliados, basicamente correlacionam a contagem sérica de plaquetas com o grau de fibrose hepática nos pacientes com hepatite crônica C. Assim, recentemente WAI et al. ${ }^{(47)}$, avaliando a histologia hepática de pacientes com hepatite crônica $\mathrm{C}$, observaram que os pacientes plaquetopênicos têm maior grau de fibrose hepática, sugerindo que a mesma seria fator preditivo de fibrose hepática. BONACINI et al. ${ }^{(3)}$, utilizando um escore composto por níveis séricos de plaquetas, índice ALT/AST e tempo de protrombina, obtiveram correlação significativa para a fibrose avançada e cirrose. POYNARD et al. ${ }^{(34)}$ apresentaram resultados positivos, correlacionando número de plaquetas e idade do paciente com o grau de fibrose hepática. Com esses achados, poder-se-ia inferir que quanto maior o grau de fibrose, maior seria a disfunção hepatocelular e, conseqüentemente, menor seria a contagem sérica de plaquetas. Os achados desta casuística demonstraram uma tendência de associação entre a plaquetopenia e o grau de disfunção hepatocelular, sendo a média da contagem de plaquetas de $99.200 / \mathrm{mm}^{3}$ nos pacientes Child C e $206.224 / \mathrm{mm}^{3}$ nos Child A, sem no entanto atingir valores estatisticamente significativos.

Dada a sugestão de que tanto a hipertensão portal, quanto o grau de falência hepatocelular contribuiriam para a plaquetopenia observada nos pacientes com hepatopatia crônica, avaliaram-se ambos os fatores, correlacionando-os com o número de plaquetas.

Assim, ao se agrupar os pacientes Child A com GPVH menor que $12 \mathrm{~mm} \mathrm{Hg}$ e os pacientes Child C com GPVH maior que $12 \mathrm{~mm} \mathrm{Hg}$, verificou-se a tendência no número médio de plaquetas ser menor no segundo grupo. O fato de não se ter encontrado diferença estatística pode estar na dependência do número de casos de cada grupo, bem 
como de se ter observado razoável variabilidade no número de plaquetas dentro dos mesmos. É possível que se maior número de pacientes fosse avaliado, alcançar-se-ia diferença estatística. Ainda, De NORONHA et al. ${ }^{(13)}$, em estudo avaliando 92 pacientes com doença hepática crônica, concluíram que a gravidade da hepatopatia é fator determinante do grau de plaquetopenia, sugerindo como mecanismos principais tanto o hiperesplenismo (pela hipertensão portal), quanto a diminuição da trombopoietina (pela redução da síntese hepática) ${ }^{(13)}$.

\section{CONCLUSÃO}

A despeito do presente estudo não ter conseguido demonstrar, de forma significativa, a associação entre a plaquetopenia e o grau de hipertensão portal, bem como com o grau de disfunção hepatocelular, pelas tendências observadas, podemos inferir que ambos os fatores estejam implicados no baixo nível de plaquetas, conforme sugerido por outros autores ${ }^{(13,21,22,32,39)}$.

Dittrich S, Mattos AA, Cheinquer H, Araújo FB. Correlation between platelet blood levels and the hepatic venous pressure gradient among patients with cirrhosis. Arq Gastroenterol 2005;42(1):35-40.

ABSTRACT - Background - Determination of hepatic venous pressure gradient is the main method used to assess portal pressure. Recently, platelet blood levels has been indicated as a non-invasive marker of the presence of portal hypertension. Aim - To correlate platelet blood levels with the hepatic venous pressure gradient among patients with cirrhosis. Patients and Methods - A total of 83 cirrhotic patients who had undergone hepatic venous pressure gradient over the last 6 years were included. Patients were divided in groups according to Child-Pugh classification. All had upper digestive endoscopy to assess the presence of esophageal varices and platelet serum levels were recorded. Results - Platelet serum levels range varied between $45,000 / \mathrm{mm}^{3}$ and 389,000/mm $(\mathrm{mean}$ : 104,099; standard deviation: 58,776). Mean hepatic venous pressure gradient was $15.2 \mathrm{~mm} \mathrm{Hg}$ with a standard deviation of $6.4 \mathrm{~mm} \mathrm{Hg}$ (range: 1 to $29 \mathrm{~mm}$ $\mathrm{Hg}$ ). Simple linear regression analysis was applied to verify an association of hepatic venous pressure gradient and platelet serum levels, revealing a weak correlation between both variables. We observed a progressive reduction of serum platelet levels as esophageal varices diameter increased and hepatocellular function (established by Child-Pugh classification) decreased. However, these findings did not reach statistical significance. Conclusion - Despite the lack of a statistical significant correlation among serum platelet levels and hepatic venous pressure gradient or hepatocellular function, there was a clear tendency indicating that those variables could be involved in the pathogenesis of low platelet levels.

HEADINGS - Portal pressure. Hypertension, portal. Platelet count. Liver cirrhosis.

\section{REFERÊNCIAS BIBLIOGRÁFICAS}

1. Armonis A, Patch D, Burroughs A. Hepatic venous pressure measurement: an old test as a new prognostic marker in cirrhosis? Hepatology 1997;25:245-8.

2. Bean WB, Paul WD, Franklin M. Preliminary studies on an indirect method for determining portal pressure. J Clin Invest 1949;28:769-70.

3. Bonacini M, Hadi G, Govindarajan S, Lindsay KL. Utility of a discriminant score for diagnosing advanced fibrosis or cirrhosis in patients with chronic hepatitis $\mathrm{C}$ virus infection. Am J Gastroenterol 1997;92:1302-4.

4. Bosch J, Navasa M, Garcia-Pagán JC, Pizcueta MP, Rodés J. Portal hypertension. Med Clin North Am 1989;73:931-53.

5. Bosch J. Prevention of variceal rebleeding: endoscopes, drugs and more. Hepatology 2000;32:660-1.

6. Brazzini A, Hunter DW, Darcy MD, Smith TP, Cragg AH, Castaneda-Zuniga WR, Amplatz K. Safe splenoportography. Radiology 1987;162:607-9.

7. Bruix J, Castells A, Bosch J, Feu F, Fuster J, Garcia-Pagan JC, Visa J, Bru C, Rodes J. Surgical resection of hepatocellular carcinoma in cirrhotic patients: prognostic value of preoperative portal pressure. Gastroenterology 1996;111:1018-22.

8. Burcharth F, Rasmussen SN. Localization of the porta hepatis by ultrasonic scanning prior to percutaneous transhepatic portography. Br J Surg 1974;47:598-602.

9. Burcharth F, Joyce F. Percutaneous transhepatic catheterization of the portal venous system. J Gastroenterol Hepatol 1987;2:569-87.

10. D'amico G, Pagliaro L, Bosch J. The treatment of portal hypertension: a meta-analytic review. Hepatology 1995;22:332-48.

11. Darcy MD, Kanterman RY, Kleinhoffer MA, Vesely TM, Picus D, Hicks ME, Pilgram TK. Evaluation of coagulation tests as predictors of angiographic bleeding complications. Radiology 1996;198:741-4.

12. De Francis R. Updating consensus in portal hypertension: report of the Baveno 11 consensus workshop on definitions, methodology and therapeutic strategies in portal hypertension. J Hepatol 2000;33:846-52.

13. De Noronha R, Taylor BA, Wild G, Triger DR, Greaves M. Inter-relationships between platelet count, platelet IgG, serum IgG, immune complexes and the severity of liver disease. Clin Lab Haematol 1991;13:127-35.

14. Fenyves D, Pomie-Layrargues G, Willems B, Côté J. Intrahepatic pressure measurement: not an accurate reflection of portal vein pressure. Hepatology 1988;8:211-6.

15. Garcia-Tsao G, Groszmann RJ, Fischer RL, Conn HO, Atterbury CE, Glickman M. Portal pressure, presence of gastroesophageal varices and variceal bleeding. Hepatology $1985 ; 5: 419-24$
16. George JN. Platelets. Lancet 2000;355:1531-9.

17. Gertsch P, Fischer G, Kleber G. Manometry of esophageal varices: comparison of an endoscopic baloon technique with needle puncture. Gastroenterology 1993;105:1159-66.

18. Grace ND, Groszmann RJ, Garcia-Tsao G, Burroughs AK, Pagliaro L, Makuch RW, Bosch J, Stiegmann GV, Henderson JM, De Franchis R, Wagner JL, Conn HO, Rodes J. Portal hypertension and variceal bleeding: an AASLD single topic symposium. Hepatology 1998;28:868-80.

19. Groszmann RJ, Glickman M, Blei AT, Storer E, Conn HO. Wedged and free hepatic vein venous pressure measured with balloon catheter. Gastroenterology 1975;76:253-8.

20. Groszmann RJ, Atterbury LE. The pathophysiology of portal hypertension: a basis for classification. Semin Liver Dis 1982;2:217-86

21. Kawasaki T, Takeshita A, Souda K, Kobayashi Y, Kikuyama M, Suzuki F, Kageyama F, Sasada Y, Shimisu E, Murohisa G, Koide S, Yoshimi T, Nakamura H, Ohno R. Serum thrombopoietin levels in patients with chronic hepatitis and liver cirrhosis. Am J Gastroenterol 1999;94:1918-22.

22. Kitanok T, Shimodaira S, Ito T. Liver cirrhosis with marked thrombocytopenia and highly evaluated serum thrombocytopenia levels. Int J Hematol 1999;70:52-5.

23. Le Moine O, Hadengue A, Moreau R, Sogni P, Soupison T, Yang S, Hartleb M, Lebrec D. Relationship between portal pressure, esophageal varices, and variceal bleeding on basis of stage and cause of cirrhosis. Scand J Gastroenterol 1997;32:731-5.

24. Lebrec D, Benhamou JP. Noncirrhotic intrahepatic portal hypertension. Semin Liver Dis $1986 ; 6: 332-40$

25. Lechner K, Niessner H, Thaler E. Coagulation abnormalities in liver disease. Semin Thromb Hemost 1977;4:40-56.

26. MacMathuna P, Westaby D, Williams R. Portal hypertension: pathophysiology, diagnosis and treatment. In: Millward-Sadler GH, Wright R, Arthur MJP, editors. Wright's liver and biliary disease: pathophysiology, diagnosis and management. $3^{\text {rd }}$ edition. London: Saunders; 1992. p.1296-322.

27. Merkel C, Bolognesi M, Bellon S, Zuin R, Noventa F, Finucci G, Sacerdoti D, Angeli P, Gatta A. Prognostic usefulness of hepatic vein catheterization in patients with cirrhosis and esophageal varices. Gastroenterology 1992;102:973-9.

28. Myers JD, Taylor WJ. An estimation of portal venous pressure by occlusive catheterization of a hepatic venule. J Clin Invest 1951;30:662-3.

29. The North Italian Endoscopic Club for Study and Treatment of Esophageal Varices. Prediction of the first variceal hemorrhage in patients with cirrhosis of the liver and esophageal varices. A prospective multicenter study. N Engl J Med 1988;319:983-9.

30. Okuda K, Takayasu K, Iwamoto S. Angiography in the diagnosis of the liver disease. Semin Liver Dis 1989;9:50-62. 
31. Pascal JP, Cales P. Propranolol in the prevention of first upper gastrointestinal tract hemorrhage in patients with cirrhosis of the liver and esophageal varices. N Engl J Med 1987;317:856-61.

32. Peck-Radosavlievic M, Zacherl J, Meng YG, Pidlich J, Lipinski E, Langle F, Steininger $\mathrm{R}$, Muhlbacher F, Gangl A. Is inadequate thrombopoietin production a major cause of thrombocytopenia in cirrhosis of the liver? J Hepatol 1997;27:127-31.

33. Peck-Radosavljevic M. Thrombocytopenia in liver disease. Can J Gastroenterol 2000;14 (Suppl D):60D-6D.

34. Poynard T, Bedossa P. Age and platelets count: a simple index for predicting the presence of histological lesions in patients with antibodies to hepatitis $\mathrm{C}$ virus. Metavir and Clinivir Cooperative Study Groups. J Viral Hepat 1997;4:199-208.

35. Pugh RN, Murray-Lyon IM, Dawson JL. Transection of the oesophagus of bleeding oesophageal varices. Br J Surg 1973;60:646-9

36. Sabbá C, Merkel C, Zoli M. Interobserver and interequipment variability of echoDoppler examination of the portal vein: effect of a cooperative training program. Hepatology 1995;21:428-33.

37. Schepis $\mathrm{F}$, Cammà $\mathrm{C}$, Niceforo $\mathrm{D}$. Which patients with cirrhosis should undergo endoscopic screening for esophageal varices detection? Hepatology 2001;33:333-8.

38. Sherlock S, Dooley J. The portal venous system and portal hypertension. In: Sherlock S, Dooley J. Diseases of the liver and biliary system. $10^{\text {th }}$ ed. Oxford: Blackwell Science; c1997. p.135-80

39. Shimodaira S, Ishida F, Ishikawa N, Tahara T, Kato T, Kodaira H, Ito T, Tanaka E, Sodeyama T, Kiyosawa K, Kitano K. Serum thrombopoietin (c - Mpl Ligand) levels in patients with liver cirrhosis. Thromb Haemost 1996;76:545-8.

40. Siringo S, Bolondi L, Gaiani S. Timing of the first variceal hemorrhage in cirrhotic patients: prospective evaluation of Doppler flowmetry, endoscopy and clinical parameters. Hepatology 1994;20:66-73.

41. Stanley AJ, Robinson I, Forrest EH, Jones AL, Hayes PC. Haemodynamic parameters predicting variceal haemorrhage and survival in alcoholic cirrhosis. Q J Med 1998;91:19-25.

42. Taylor WJ, Myers JD. Occlusive hepatic venous catheterization in the study of the normal liver, cirrhosis of the liver and noncirrhotic portal hypertension. Circulation 1956;13:368-80.
43. Thomopoulos KC, Labrapoulou-Karatza C, Mimidis KP, Katsakoulis EC, Iconomou G, Nikolopoulou VN. Non-invasive predictors of the presence of large oesophageal varices in patients with cirrhosis. Dig Liver Dis 2003;35:473-8.

44. Viallet A, Marleau D, Huet M. Hemodynamic evaluation of patients with intrahepatic portal hypertension. Relationship between bleeding varices and the portohepatic gradient. Gastroenterology 1975;69:1297-300.

45. Villanueva C, Miñana J, Ortiz J, Gallego A, Soriano G, Torras X, Sainz S, Boadas J, Cusso X, Guarner C, Balanzo J. Endoscopic compared with combined treatment with nadolol and isosorbide mononitrate to prevent recurrent variceal bleeding. $\mathrm{N}$ Engl J Med 2001;345:647-55.

46. Vorobioff J, Groszmann RJ, Picabea E, Gamen M, Villavicencio R, Bordato J, More I, Audano M, Tanno H, Lerner E, Passamonti M. Prognostic value of hepatic venous pressure gradient measurements in alcoholic cirrhosis: a 10 years prospective study. Gastroenterology 1996;111:701-9.

47. Wai CT, Greenson JK, Fontana RJ, Kalbfleisch JD, Marrero JA, Conjeevaram HS, Lok ASF. A simple noninvasive index can predict both significant fibrosis and cirrhosis in patients with chronic hepatitis C. Hepatology 2003;38:518-26.

48. Westaby S, Wikinson SP, Warren R, Williams R. Spleen size and portal hypertension in cirrhosis. Digestion 1978;17:63-8.

49. Zaman A, Hapke R, Flora K, Rosen HR, Benner K. Factors predicting the presence of esophageal or gastric varices in patients with advanced liver disease. Am J Gastroenterol 1999;94:3292-6.

50. Zoli M, Iervese T, Bellon S, Merkel C. Prognostic significance of portal hemodynamics in patients with compensated cirrhosis. J Hepatol 1993;17:56-61. 